
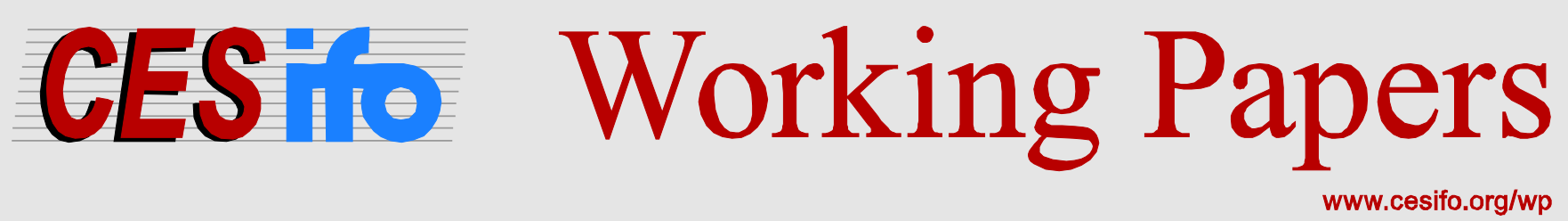

\title{
Estimating General Equilibrium Trade Policy Effects: GE PPML
}

\author{
James E. Anderson \\ Mario Larch \\ Yoto V. Yotov
}

CESIFO WORKING PAPER NO. 5592

CATEGORY 8: TRADE POLICY

NOVEMBER 2015

An electronic version of the paper may be downloaded

- from the SSRN website:

- from the RePEc website:

- from the CESifo website:

WwW.SSRN.com

www.RePEc.org

www.CESifo-group.org/wp 


\title{
Estimating General Equilibrium Trade Policy Effects: GE PPML
}

\begin{abstract}
We develop a simple estimation procedure for general equilibrium (GE) comparative static analysis of gravity models. Non-linear solvers of estimated models are replaced by (constrained) regressions. Applied economists can more readily generate results, with more intuition about the working of the model. We illustrate with a worldwide border removal application using the Poisson Pseudo-Maximum-Likelihood (PPML) estimator in STATA, iterated to deliver conditional and full general equilibrium responses. The method works by fully exploiting the combined properties of structural gravity and PPML. Our procedures readily extend to a wide class of general equilibrium production models.
\end{abstract}

JEL-Codes: F100, F140, F160.

Keywords: structural gravity, general equilibrium effects, counterfactuals, estimation.

\author{
James E. Anderson \\ Boston College \\ USA - Chestnut Hill, MA 02467 \\ james.anderson@bc.edu
}

\author{
Mario Larch \\ University of Bayreuth \\ Germany - 95440 Bayreuth \\ mario.larch@uni-bayreuth.de
}

\author{
Yoto V. Yotov \\ Drexel University \\ USA - 19072 Philadelphia PA \\ yotov@drexel.edu
}

November 9, 2015

We thank João Santos Silva and seminar participants at the Economic Research and Statistics Division of the WTO and at the ifo institute, and conference participants at the 2015 European Trade Study Group meetings (Paris) and the Panel Data Workshop on International Trade (Amsterdam) for useful comments and suggestions. Yotov is grateful to the members of the CESifo group for their hospitality during his stay at the ifo Insitute in 2015. 


\section{Introduction}

We derive an estimator of the comparative statics of gravity models in conditional general equilibrium, the Modular Trade Impact of Head and Mayer (2014). Our innovation is to infer rather than solve for changes in multilateral resistances as trade costs or endowments are counterfactually changed. An extension estimates the full general equilibrium impact when endowments are fixed but sellers' prices change (Head and Mayer (2014)'s General Equilibrium Trade Impact), such as in the Ricardian Eaton-Kortum model (Eaton and Kortum, 2002 ) and the Armington-CES model of Anderson and van Wincoop (2003). Multi-sector applications beyond the scope of this paper can similarly use estimated Modular Trade Impacts for each sector that nest in any compatibly separable inter-sectoral general equilibrium production model. This includes most applied general equilibrium models.

Structural gravity implies that the ratio of predicted bilateral trade to its benchmark frictionless flow is equal to a power transform of the ratio of bilateral trade cost to the product of inward and outward multilateral resistances. Multilateral resistances can be inferred from origin and destination fixed effects in a standard gravity regression along with the more familiar inference of unobservable bilateral trade costs. The multilateral resistances also are the solution to a non-linear pair of equation systems derived under the theoretical assumptions from global market clearance for each country's sales and meeting each country's budget constraint. Fally (2015) shows that when gravity is estimated with Poisson Pseudo-Maximum-Likelihood (PPML), the estimated fixed effects are exactly equal to the multilateral resistances that satisfy the equation system. Our method rests on this result.

A typical counterfactual comparative static exercise using gravity is to change some bilateral friction, e.g. remove a tariff, and then calculate the effects on trade flows and other variables of interest. The partial effect is based on the estimated bilateral friction, e.g. the percentage reduction in buyers' price times the trade elasticity. The Modular Trade Impact requires the new counterfactual multilateral resistances, typically solved from the equation system with a nonlinear solver. Our alternative method is to re-estimate with the 
counterfactual friction(s) using PPML. This is a more readily accessible way to generate the general equilibrium comparative statics of gravity models. Another benefit is the combination of statistical with economic theoretic intuition in interpreting results. The estimated fixed effects (and their changes) provide traditionally strong fit to the data (under the PPML structure) along with satisfying equilibrium market clearance and budget constraints.

We demonstrate the success and effectiveness of the proposed estimation GE methods by focusing on a hypothetical scenario that removes all international borders to trade in manufactures for 40 countries and a rest-of-world aggregate over the period 1990-2002. Our estimates suggest that, all else equal, international borders decrease trade by an average of 79 percent (std.err. 2.575). More importantly for the purposes of this study, we use the estimates of the fixed effects changes to construct general equilibrium changes in the multilateral resistances, total exports, and real consumption for each of the countries in our sample. Welfare changes range between 5 and 40 percent and are concentrated in the smaller and less developed economies in our sample. We demonstrate as a check on our method that these indexes are identical to the corresponding numbers obtained by solving the non-linear gravity system in Matlab.

Our methods complement and extend the 'exact hat algebra' methods of Dekle, Eaton and Kortum (2008).1 We differ quantitatively from the usual applications of Dekle, Eaton and Kortum (2008) in basing calculations on fitted (predicted) trade flows rather than observed trade flows, with the presumed advantage of controlling for measurement error in the trade flow data. We differ computationally as described above, but our simple estimation procedure delivers identical results.

An important potential advantage of our approach is the generation of bootstrap standard errors for comparative static general equilibrium exercises. The attractive econometric properties of gravity and the useful sufficient statistic property of multilateral resistance readily suggest the bootstrap approach to giving comparative statics a statistical measure

\footnotetext{
1 Head and Mayer (2014) provide Stata code solving the multilateral resistance terms via contraction mapping for use in such exercises. Costinot and Rodríguez-Clare (2014) review the related literature.
} 
of reliability that is lacking in current counterfactual methods. We discuss briefly some problems to be solved before realization of this promise of bootstrapping in future work.

The rest of the paper is organized as follows. Section 2 reviews the structural gravity model and its empirical treatment and discusses the relationship between the structural gravity terms and the corresponding fixed effects from the empirical gravity equation. Section 3 describes our simple 3-step estimation procedure to obtain estimates of the general equilibrium effects of trade policy and changes in trade costs with the PPML estimator. In Section 4 we simulate a hypothetical scenario that removes all international borders to trade in order to establish the effectiveness and the empirical relevance of our methods. Section 5 discusses possible extensions of our methods beyond a one sector economy. Section 6 concludes. Finally, the Appendix includes a sample code that implements our procedures.

\section{Structural Gravity with Fixed Effects}

Anderson (1979) derives the first structural gravity model of trade under the assumptions of identical Constant Elasticity of Substitution (CES) preferences across countries for national varieties differentiated by place of origin (Armington, 1969): $:^{2}$

$$
\begin{aligned}
X_{i j} & =\left(\frac{t_{i j}}{\Pi_{i} P_{j}}\right)^{1-\sigma} Y_{i} E_{j}, \\
P_{j}^{1-\sigma} & =\sum_{i}\left(\frac{t_{i j}}{\Pi_{i}}\right)^{1-\sigma} Y_{i}, \\
\Pi_{i}^{1-\sigma} & =\sum_{j}\left(\frac{t_{i j}}{P_{j}}\right)^{1-\sigma} E_{j}, \\
p_{j} & =\frac{Y_{j}^{\frac{1}{1-\sigma}}}{\gamma_{j} \Pi_{j}} .
\end{aligned}
$$

Here, $X_{i j}$ denotes the value of shipments at destination prices from region of origin $i$ to

\footnotetext{
2 Anderson (2011) and Costinot and Rodríguez-Clare (2014) offer discussions of alternative microfoundations for structural gravity. Allen, Arkolakis and Takahashi (2014) discuss the uniqueness and existence properties of a wide class of structural gravity models.
} 
region of destination $j$. The order of double subscripts denotes origin to destination. $E_{j}$ is the expenditure at destination $j$ from all origins and $Y_{i}$ denotes the sales at destination prices from $i$ to all destinations. $t_{i j} \geq 1$ denotes the variable trade cost factor on shipments of goods or services from $i$ to $j$, and $\sigma$ is the elasticity of substitution across varieties.

$P_{j}$ is the inward multilateral resistance (IMR), which aggregates the incidence of trade costs on consumers in each country, and also the CES price index of the demand system. $\Pi_{i}$ is the outward multilateral resistance (OMR), which from (3) aggregates $i$ 's outward trade costs relative to destination price indexes. Multilateral resistance is a conditional general equilibrium concept, since $\left\{\Pi_{i}, P_{j}\right\}$ solve equations (2)-(3) for given $\left\{Y_{i}, E_{j}\right\}$. Note also that (2)-(3) solves for $\left\{\Pi_{i}, P_{j}\right\}$ only up to a scalar. If $\left\{\Pi_{i}^{0}, P_{j}^{0}\right\}$ is a solution then so is $\left\{\lambda \Pi_{i}^{0}, P_{j}^{0} / \lambda\right\}$. Therefore, a normalization of one of the multilateral resistances is needed in order to obtain a unique solution for $(2)-(3) t^{3}$

Finally, equation (4) is derived from the market clearance:

$$
Y_{i}=\sum_{j} X_{i j}=\sum_{j}\left(\gamma_{i} p_{i} t_{i j} / P_{j}\right)^{1-\sigma} E_{j}=\left(\gamma_{i} p_{i}\right)^{1-\sigma} \sum_{j}\left(t_{i j} / P_{j}\right)^{1-\sigma} E_{j} \quad \text { for all } \quad j
$$

where $p_{i}$ is the exporter's supply price of country $i$ and $\gamma_{i}$ is a positive distribution parameter of the CES utility function. Using Equation (3) leads to Equation (4).

Gravity equations are recommended to be estimated with importer and exporter fixed effects by Feenstra (2004), a recommendation followed by most of the subsequent literature. In addition, many recent papers follow the recommendation of Santos Silva and Tenreyro (2006) who argue in favor of the PPML estimator for gravity regressions in order to account for heteroskedasticity and to take advantage of the information contained in the zero trade flows. Taking these considerations into account, many recent studies employ a version of the following empirical gravity model:

$$
X_{i j}=\exp \left(\mathbf{T}_{i j} \boldsymbol{\beta}+\pi_{i}+\chi_{j}\right)+\epsilon_{i j}
$$

\footnotetext{
${ }^{3}$ See Anderson and Yotov (2010) for detailed discussions of the multilateral resistances.
} 
Here, $\mathbf{T}_{i j}$ is the vector of trade cost variables, $\boldsymbol{\beta}$ is a vector of coefficients, $\epsilon_{i j}$ is an error term, $\pi_{i}$ is an exporter fixed effect that accounts for the outward multilateral resistances and for outputs, and $\chi_{j}$ is an importer fixed effect that accounts for expenditures and for the inward multilateral resistances $4^{4}$ To avoid perfect collinearity, we either have to drop one exporter and one importer fixed effect or one fixed effect and the constant. Our choice is to drop one importer fixed effect, $\chi_{0}$, and the constant, implying that all other fixed effects are identified relative to $\chi_{0}$. Further, note that solving the system (2)-(3) requires normalizing one of the multilateral resistances. By choice, we normalize the multilateral resistance that corresponds to the dropped importer fixed effect, $\widehat{P}_{0}=1$. With the normalized $\widehat{P}_{0}=1$, the theoretical interpretation of the importer fixed effect $\widehat{\chi}_{0}$ is $E_{0}$, but since it is dropped, $\widehat{\chi}_{0}=0$. Then, the theoretical interpretation of all other fixed effects is relative to $E_{0}$.

Fally (2015) demonstrates that the PPML estimates of the fixed effects from gravity estimations are perfectly consistent with the structural gravity terms. (See his Proposition 1.) Taking into account the normalization that we just discussed, this implies that the OMRs and IMRs can be recovered from the fixed effects as follows:

$$
\widehat{\Pi_{i}^{1-\sigma}}=E_{0} Y_{i} \exp \left(-\widehat{\pi}_{i}\right)
$$

and

$$
\widehat{P_{j}^{1-\sigma}}=\frac{E_{j}}{E_{0}} \exp \left(-\widehat{\chi}_{j}\right)
$$

where $\widehat{\pi}_{i}$ and $\widehat{\chi}_{j}$ are the estimated fixed effects from Equation (6), and $Y_{i}, E_{j}$ and $E_{0}$ are data. We capitalize on this property of PPML in the next section, where we also exploit the full structure of system (11)-(4) in order to develop our general equilibrium estimation method.

\footnotetext{
${ }^{4}$ With panel data, the directional fixed effects should also be time-varying.
} 


\section{GE PPML: Estimating the GE Effects of Trade Policy}

This section describes our simple 3-step estimation procedure to obtain estimates of the general equilibrium effects of trade policy with the PPML estimator.

- Step 1: 'Baseline' Scenario. This step delivers the 'Baseline' estimates and 'Baseline' GE indexes and consists of two sub-steps:

Step 1.a: Estimate 'Baseline' Gravity. Use the PPML estimator to estimate gravity with exporter and importer fixed effects:

$$
X_{i j}=\exp \left(\mathbf{T}_{i j} \boldsymbol{\beta}+\pi_{i}+\chi_{j}\right)+\epsilon_{i j}
$$

We chose PPML as our preferred estimator in this step for consistency with the rest of our procedure and due to its appealing properties for gravity estimations (see Santos Silva and Tenreyro, 2006, 2011). However, we note that any estimator can be employed to obtain the estimates of the trade cost elasticities $\boldsymbol{\beta}$ in a preliminary step.$^{5}$ In fact, the $\boldsymbol{\beta}$ 's can even be borrowed from other studies as is routinely done in the literature. In case the estimates of the trade cost elasticities are obtained externally or with another estimator than PPML, Step 1.a should be repeated with the external elasticity parameters or the obtained parameters from the estimator of your choice imposed as constraints in the PPML estimation (9):

$$
X_{i j}=\exp \left(\mathbf{T}_{i j} \overline{\boldsymbol{\beta}}+\pi_{i}+\chi_{j}\right)+\epsilon_{i j}
$$

where $\overline{\boldsymbol{\beta}}$ denotes the constrained set of trade cost coefficients. In addition, we note that Step 1.a can be used to generate a whole distribution of bootstrapped trade cost elasticity parameters that can be fed into the next steps in order to generate

\footnotetext{
${ }^{5}$ We refer the reader to Head and Mayer (2014) for informative discussions of the relative merits and caveats of PPML versus other nonlinear estimators in the gravity context.
} 
confidence intervals for the GE indexes of interest, as is for example done by Anderson and Yotov (2015). The reason is that PPML is the only estimator where the sum of fitted values of GDPs and expenditures is equal to the sum of observed values of GDPs and expenditures (see Proposition 2 of Fally, 2015), a property needed in order to guaranty that the fixed effects from gravity estimations are perfectly consistent with the structural gravity terms. In Steps 2 and 3 below, we capitalize on this PPML property and we extend Fally's analysis to estimate general equilibrium effects of changes in trade costs with the structural gravity model.

Furthermore, in principle, our approach can be used to deliver standard errors for the indexes of interest directly from the estimates of the gravity fixed effects. However, as only recently the consistency of the model parameter estimates in nonlinear panel models with two types of fixed effects has been shown by Fernández-Valz and Weidner (2015), we leave this for future research.

Step 1.b: Construct 'Baseline' GE Indexes. Use the estimates of the fixed effects from (9) together with data on outputs and expenditures to construct the multilateral resistances according to (7)-(8), where, by construction, $Y_{i}=\sum_{j} X_{i j}$ and $E_{j}=\sum_{i} X_{i j}$. Construct any other baseline GE indexes of interest (e.g. predicted exports, $\left.\sum_{j \neq i} \widehat{X}_{i j}, \forall i\right)$.

Note that in order to be able to perform counterfactual analysis, we need values for all inward and outward multilateral resistance terms, in addition to the inward multilateral resistance which is normalized. This is only possible if, after dropping one importer fixed effect and the constant, PPML does not drop any additional fixed effects or observations. If additional fixed effects are dropped, one may need to check and adjust the data in order to avoid the dropping of fixed effects by PPML. For example, one may drop countries with zero reported exports or imports to all trading partners. 
- Step 2: 'Conditional' Scenario. This step delivers the 'Conditional' gravity estimates and 'Conditional' GE indexes, which allow for changes in the IMRs and OMRs in response to changes in trade costs, but do not take output and expenditure changes into account. Again, this step consists of two sub-steps:

Step 2.a: Estimate 'Conditional' Gravity. Re-define the policy variable(s) of interest to reflect any desired trade policy changes and use PPML to estimate:

$$
X_{i j}=\exp \left(\mathbf{T}_{i j}^{c} \widehat{\boldsymbol{\beta}}+\pi_{i}^{c}+\chi_{j}^{c}\right)+\epsilon_{i j}^{c}
$$

Here $\mathbf{T}_{i j}^{c}$ is the vector of counterfactual trade policy covariates. For example, an indicator for Regional Trade Agreements (RTAs) can be amended to eliminate an existing agreement or to introduce a new one: ${ }^{6}$ the hat on $\boldsymbol{\beta}$ indicates the fact that the trade cost coefficients are constrained to the estimated values from the baseline specification (9); and the superscript $c$ denotes counterfactual variables. Notice that the data remains the same in this counterfactual exercise: $X_{i j}$ remains the same and thus so do $Y_{i}$ and $E_{j}$. The experiment infers the fixed effects (multilateral resistances) that are consistent with the original data with the counterfactual trade costs $\mathbf{T}_{i j}^{c}$.

This step can be implemented directly in Stata (StataCorp LP, 2013) using version 2.2.2 (October 10th, 2015) or newer of 'ppml' command of Santos Silva and Tenreyro (2006) with the 'offset()' option to implement the counterfactual scenario as a constraint on the gravity trade costs, i.e.,

$$
\operatorname{ppml} X_{i j} \pi_{i}^{c} \chi_{j}^{c} \text {, noconst offset }\left(\mathbf{T}_{i j}^{c} \widehat{\boldsymbol{\beta}}\right)
$$

To obtain the latest version of the 'ppml' command just type 'ssc install ppml' in Stata. Appendix 6 includes a code that implements Steps 1 through 3 in Stata StataCorp 
LP, 2013). The full Stata codes accompanying this paper are available upon request.

Step 2.b: Construct 'Conditional' GE Indexes. Repeat Step 1.b with the new fixed effects estimates from (11) and the original data on outputs and expenditures to construct the 'Conditional' GE (the Modular Trade Impact of Head and Mayer, 2014) estimates of the multilateral resistances and construct any other GE indexes of interest. The differences, in percentage, between the baseline indexes from Step 1.b and the counterfactual indexes from this step measure the 'Conditional' GE effects of the simulated trade policy. Specifically, the percentage change in welfare in the 'Conditional' GE scenario can be calculated by the change in real GDP, i.e. 7

$$
\widehat{W}_{i}=\frac{Y_{i}^{c} / \hat{P}_{i}^{c}}{Y_{i} / \hat{P}_{i}}=\frac{\hat{P}_{i}}{\hat{P}_{i}^{c}}, \forall i
$$

where moving from the middle to the rightmost equality recognizes that output is kept exogenous in the 'Conditional' scenario, i.e. $Y_{i}^{c}=Y_{i}$.

Note that we obtain power transforms of the inward multilateral resistances according to Equation (8). Therefore, to construct real GDP, we use a standard value for the elasticity of substitution $\sigma=7$. In principle, $\sigma$ can also be estimated directly from an empirical gravity model that includes as a covariate any direct price shifter, e.g. tariff. See for an overview of varies ways to obtain estimates for the elasticity of substitution Head and Mayer (2014).

- Step 3: 'Full Endowment' Scenario. This step delivers the 'Full Endowment' gravity estimates and 'Full Endowment' GE indexes, which in addition to changes in the IMRs and OMRs capture changes in output and expenditure. Also Step 3 consists of two sub-steps:

\footnotetext{
${ }^{7}$ Note that due to our normalization of $P_{0}=1$, welfare changes in the 'Conditional' scenario are relative to reference country 0 .
} 
Step 3.a: Estimate 'Full Endowment' Gravity. Allow for endogenous response in the value of outputs/incomes and expenditures, which are given by $Y_{i}^{c}=\left(p_{i}^{c} / p_{i}\right) Y_{i}$ and $E_{i}^{c}=\left(p_{i}^{c} / p_{i}\right) E_{i}$ in an endowment economy where trade imbalance ratios $\phi_{i}=E_{i} / Y_{i}$ are assumed to stay constant in the counterfactual for each country $i$ (allowing for balanced trade as a special case). The endogenous changes in output/income and expenditure will trigger additional changes in the multilateral resistance (MR) terms and so forth. As the PPML estimator with the appropriate fixed effects ensures that the sum of fitted values of GDPs and expenditures is equal to the sum of observed values of GDPs and expenditures, changes in output/income and expenditure cannot be directly estimated in one step with PPML. Therefore, we use the structural gravity Equation (1) to translate the changes in output and expenditure, triggered by the changes in factory-gate prices, into changes in trade flows:

$$
X_{i j}^{c}=\frac{\left(t_{i j}^{1-\sigma}\right)^{c}}{t_{i j}^{1-\sigma}} \frac{Y_{i}^{c} E_{j}^{c}}{Y_{i} E_{j}} \frac{\Pi_{i}^{1-\sigma} P_{j}^{1-\sigma}}{\left(\Pi_{i}^{1-\sigma}\right)^{c}\left(P_{j}^{1-\sigma}\right)^{c}} X_{i j},
$$

where $t_{i j}^{1-\sigma}=\exp \left(\mathbf{T}_{i j} \boldsymbol{\beta}\right)$ and $\left(t_{i j}^{1-\sigma}\right)^{c}=\exp \left(\mathbf{T}_{i j}^{c} \widehat{\boldsymbol{\beta}}\right)$. The new values for outputs and expenditures, $Y_{i}^{c}$ and $E_{j}^{c}$, are obtained by using the market clearing conditions $p_{i}=\left(\frac{Y_{i}}{Y}\right)^{\frac{1}{1-\sigma}} \frac{1}{\gamma_{i} \Pi_{i}}$ to translate the 'Conditional' GE effects on the MR terms into 'firstorder' changes in factory-gate prices, i.e.

$$
p_{i}^{c} / p_{i}=\left[\exp \left(\widehat{\pi}_{i}^{c}\right) / \exp \left(\widehat{\pi}_{i}\right)\right]^{\frac{1}{1-\sigma}}
$$

As imposed in Step 1, the vector of prices $\left\{p_{i}^{c}\right\}$ is normalized by $P_{0}=\sum_{i}\left(\gamma_{i} p_{i} t_{i 0}\right)^{1-\sigma}=$ 1. Note that the changes in trade implied by Equation (14) are not the 'Full Endowment' GE changes. The reason is that they only reflect the 'Conditional' OMR changes and do not allow for immediate changes in the value of outputs. This is why we label these initial changes in the factory-gate prices and in trade 'first-order'. Thus, in effect, the methods that we represent here are an interactive estimation equivalent to 
the 'exact hat' procedures from Dekle, Eaton and Kortum (2008).

Repeat Step 2.a with the new values for trade. The idea is that, using the new values of trade, the PPML estimator will translate the initial response of factory-gate prices into changes in the gravity fixed effects, which (in combination with the new values for income $Y_{i}^{c}=\sum_{j} X_{i j}^{c}$ and expenditures $\left.E_{j}^{c}=\sum_{i} X_{i j}^{c}\right)$ can be used to obtain additional 'second-order' responses in the MR terms. Repeat Step 3.a to obtain a new set of factory-gate prices and new values of trade, income and expenditures. Iterate until convergence, e.g. until the change between two subsequent iterations in each of the factory-gate prices is smaller than a pre-defined tolerance criterion.

Step 3.b: Construct 'Full Endowment' GE Indexes. Construct 'Full Endowment' GE indexes of interest following the procedures from Step 1.b. The differences, in percentage, between the baseline indexes from Step 1.b and the counterfactual indexes from this step measure the 'Full Endowment' GE effects of the simulated trade policy. The percentage change in welfare in the 'Full Endowment' GE scenario can again be calculated by the change in real GDP, i.e.,

$$
\widehat{W}_{i}=\frac{Y_{i}^{c} / \hat{P}_{i}^{c}}{Y_{i} / \hat{P}_{i}}, \forall i
$$

Note that with balanced trade or constant shares of trade imbalances, the change in output and expenditure are identical for each country. Hence, real GDP changes correspond to changes in real expenditures. Further, Arkolakis, Costinot and RodríguezClare (2012) demonstrate that the welfare/real consumption gains from trade liberalization obtained from a wide class of trade models with alternative microfoundations can all be expressed as a combination of two sufficient statistics including intra-national trade as share of total expenditures $\left(X_{i i} / E_{i}\right)$ and the trade elasticity of substitution $(1-\sigma)$. This holds for our framework. 
These three steps can be performed with any statistic/econometrics software that is able to estimate a Poisson model and is capable of handling loops. Specifically, no non-linear equation solver is necessary. Hence, it can be easily applied by anyone working empirically.

The procedures here resemble closely the 'exact hat algebra' procedures from Dekle, Eaton and Kortum (2008). They differ quantitatively from the usual practice in using the predicted value of bilateral trade instead of the observed value of trade. Otherwise, as is well understood now, in the one good case the Armington CES endowments model is an equivalent representation of the structural gravity model. The CES parameter $1-\sigma$ is alternatively interpreted as a Frèchet distribution parameter and the sales variable $Y_{i}=p_{i} q_{i}$ is interpreted as the wage bill $w_{i} L_{i}$.

\section{Trade Without Borders. An Application of GE PPML}

In order to establish the effectiveness and the empirical relevance of our methods, we focus on a hypothetical scenario that removes all international borders to trade in manufactures for 40 countries and a rest-of-world aggregate over the period 1990-2002. The data set that we employ here is a subsample of the data from Anderson and Yotov (2015), which covers total manufacturing trade, including intra-national trade for 40 countries and a rest of the world aggregate over the period 1990-2002. Given the methodological purposes of this study, we focus on the year 2002 and we refer the reader to Anderson and Yotov (2015) for further details on the dataset. While our goal in this section is to demonstrate the success of the estimation methods that we propose, we note that our experiment of removing international borders can be interpreted as a quantification of the effects of an effective multilateral trade facilitation exercise, thus, complementing the widely used counterfactual analysis of reversal to autarky, cf. Costinot and Rodríguez-Clare (2014).

We specify a simple empirical gravity specification:

$$
X_{i j}=\exp \left(\beta_{1} \ln D I S T_{i j}+\beta_{2} C N T G_{i j}+\beta_{2} B R D R_{i j}+\pi_{i}+\chi_{j}\right)+\epsilon_{i j},
$$


where all bilateral trade costs are approximated by the logarithm of bilateral distance, $\ln D I S T_{i j}$, an indicator variable that takes a value of one when trading partners $i$ and $j$ are contiguous, $C N T G_{i j}$, and an indicator variable for international borders, $B R D R_{i j}$, which takes a value of one for international trade and it is equal to zero otherwise. Specification (17) is consistent with structural gravity due to the presence of the exporter and importer fixed effects, which account for the multilateral resistances as well as for outputs and expenditures.

Step 1.a delivers estimates of the effects of distance, contiguity and international borders:

$$
X_{i j}=\exp \left(\underset{(0.052)}{\mathbf{0 . 9 4 8}} \ln D I S T_{i j}+\underset{(0.102)}{\mathbf{0 . 4 7 8} C N T G_{i j}}-\underset{(0.122)}{\mathbf{1 . 5 5 4} B R D R_{i j}}+\widehat{\pi}_{i}+\widehat{\chi}_{j}\right)+\widehat{\epsilon}_{i j}
$$

The estimates of the standard gravity variables are in accordance with prior expectations. A negative and highly statistically significant estimate of the effect of distance $\beta_{1}=-0.948$ (std.err. 0.052) does not differ significantly from the conventional estimate of -1 . There is a positive and highly significant effect of contiguity $\beta_{2}=0.478$ (std.err. 0.102). Both estimates are readily comparable with existing indexes from the literature. See Head and Mayer (2014). This establishes the representativeness of our sample.

In addition, our estimates suggest that, all else equal, international borders decrease trade by an average of 79 percent (std.err. 2.575), calculated as $\left(\exp \left[\widehat{\beta}_{3}\right]-1\right) \times 100$ with standard errors obtained with the Delta method. The estimates from Equation (18) can be used to construct all baseline indexes of interest, as specified in Step 1.b of our procedure. Their values are suppressed for expositional ease.

Next, we follow Step 2.a to obtain 'Conditional' GE estimates that correspond to the removal of international borders:

$$
X_{i j}=\exp \left(-0.948 \ln D I S T_{i j}+0.478 C N T G_{i j}+\pi_{i}^{c}+\chi_{j}^{c}\right)+\epsilon_{i j}^{c}
$$

Here, we have constrained the estimates on the trade cost covariates to their baseline values 
and we have removed the international border covariate completely, which is equivalent to keeping it with all values of the international border dummy $B R D R_{i j}$ set to zero. We use the estimates of the fixed effects and of the coefficients on the trade cost variables to construct multilateral resistances, total exports, and real consumption for each of the countries in our sample as described in Step 2.b. In addition, we obtain the same indexes by solving the non-linear gravity system in Matlab.

Figure 1 shows that the levels of the 'Conditional' GE equilibrium for the IMRs, OMRs, total exports and real GDP from the estimation in Stata and from the solution of the nonlinear gravity system (in Matlab) are identical. Figure 2 demonstrates that this is also true for the percentage changes in the GE indexes from the 'Baseline' to the 'Conditional' GE scenario. As depicted in the bottom right panel of Figure 2 , the 'Conditional' general equilibrium estimates suggest that abolishing international borders leads to welfare changes between about -7 and 14 percent relative to our reference country Germany, where real GDP changes in the 'Conditional' GE equilibrium are zero by construction. Note that the exact magnitudes depend on the size of the trade elasticity, which we set to $1-\sigma=1-7=-6$.

Finally, we follow Step 3 to obtain estimates and GE indexes of the 'Full Endowment' GE effects of the removal of international borders. Once again, we compare our Stata estimates with those obtained in Matlab. Figure 3 confirms the equivalence between the two methods concerning the level of the variables in the 'Full Endowment' GE scenario. This again holds for the percentage changes between the 'Baseline' and the 'Full Endowment' GE indexes, which are plotted in Figure 4 .

The results imply that abolishing international borders leads to welfare gains between about 4.8 and 40.3 percent, captured in the bottom right panel of Figure 4 . Overall, our findings indicate that a complete removal of international borders would benefit the most developed and large nations less than the less developed and smaller economies in our sample. The country that would gain the least is the United States, closely followed by Japan (5.6 percent). China, Korea and Germany all would register gains from the complete removal 
of international borders of less than 15 percent. Great Britain is sixth form the bottom in our list with a gain of 15.5 percent. On the other side of the spectrum we find smaller and less developed economies. The biggest winners from the complete removal of international borders include some Latin American economies. Bolivia would enjoy the largest increase in real GDP (40.3 percent). Uruguay, Ecuador, Mexico, Colombia are also in the top-10 countries according to this criteria with gains of more than 30 percent. Other big winners include some European economies, e.g. Iceland, Bulgaria, and Norway, as well Canada, Tunisia, and Morocco.

The result that more developed countries will gain less from a potential removal of international borders quantifies the familiar undergraduate textbook intuition that the smaller of two countries gains more moving from autarky to free trade, and the newer intuition from Arkolakis, Costinot and Rodríguez-Clare (2012) that larger countries with a naturally larger home market share gain less. The quantification of removing borders only includes, however, the important modifying factor of bilateral distance penalties that differ between large and small economies. For example, Europe and North America are relatively compact groups of large economies that might be expected to gain more from a given drop in border barriers. The modifying effect of distance especially shows up in relatively large gains for small European and Latin American economies.

Note that the heterogeneity in our estimates of the welfare effects is driven exclusively by general equilibrium forces, since we obtain and use a single/common estimate of the effects of international borders on international trade. Decomposing the partial equilibrium effects of international borders on trade in developed versus developing countries is beyond the scope of this paper and, arguably, our sample coverage is not well suited for this task. However, combined with the findings form other studies, e.g. Waugh (2010), that developing countries are subject to disproportionately large trade costs, our results suggest that the gap between the potential effects of multilateral/global trade liberalization would be even wider.

In analysis that is available by request we also perform a battery of robustness experi- 
ments with various alternative scenarios and specifications including decreasing and increasing distance, removing and introducing RTAs, removing and introducing contiguous borders, changing the values for the trade cost elasticities, and using alternative data samples including cross-section and panel data. All these experiments confirm the robustness of our methods and the empirical equivalence between the results from the estimating procedure that we propose here and those from a standard, but computationally intensive, procedure that requires setting and solving the structural gravity system explicitly 8

\section{Beyond the Endowment Environment}

We demonstrated how to estimate general equilibrium effects in an endowment setting, where the value of income/production is endogenous but only due to changes in factory-gate prices. (Equivalently, income in the Ricardian Eaton-Kortum setting endogenous due to changes in the wages times the endowments of labor.)

Our procedures for estimating conditional general equilibrium comparative statics apply more generally. They can nest in any general equilibrium superstructure that endogenizes the 'endowments' vector for each country while embedding inward multilateral resistances in national expenditure functions and national profit functions. Sufficient conditions include internationally identical CES tastes (for final goods) and technology (for intermediate goods) in each sector, and in the Ricardian Eaton-Kortum version an internationally common Fréchet distribution for productivity draws (with nationally distinct absolute advantage location parameters) to justify the sectoral structural gravity structures. The vector of sectoral CES final goods price indexes facing each country determines expenditure allocation across sectors in each country's upper level expenditure function. The vector of CES intermediate goods price indexes in each country partly determines intermediate goods expenditure allocation across sectors as an argument in each country's national profit (GDP) function.

\footnotetext{
${ }^{8}$ The Stata codes (implemented in Stata, StataCorp LP, 2013) and the Matlab codes (implemented in Matlab, Mathworks, 2013) are available upon request.
} 
Caliendo and Parro (2015) is an example that satisfies these restrictions in the Ricardian Eaton-Kortum case.

Another possible channel to endogenize production is via asset accumulation. For example, Anderson, Larch and Yotov (2015) combine the Armington-CES gravity model with a dynamic model of capital accumulation. Another possible channel is through labor-leisure choice. These two possibilities retain the one-good national economy.

The idea for full general equilibrium comparative statics remains essentially the same. The conditional general equilibrium gravity modules in each sector deliver multilateral resistances to the superstructure module. Reallocation of factors and of expenditure on intermediate and final goods sectoral aggregates occurs. The new values are passed back to the sectoral conditional general equilibrium modules and new multilateral resistances are generated. These are passed back to the superstructure and reallocation occurs again. The process continues until convergence.

It is possible that other algorithms may be more efficient still. But the modular structure of our suggested approach preserves insight into what is driving the ultimate equilibrium comparative statics.

Our estimating comparative statics approach readily extends to generating bootstrapped standard errors on these comparative static experiments. It moreover suggests including the interaction of the sectoral gravity modules with the general equilibrium superstructure in the generation of standard errors. Working out the statistical properties of such generated errors is posed here as an important problem for future research. What conditions guarantee consistency? For general equilibrium models richer than the endowments model, how do other sources of error get appropriate treatment? 


\section{Conclusions}

Structural gravity models are now widely used to evaluate trade and other policies related to international trade flows in the academic literature (see for an overview Head and Mayer, 2014). While their merits are by now well understood (see for a discussion Costinot and Rodríguez-Clare, 2014), the widespread applications of these methods by more applied analysts and policymakers is still lagging behind. One of the main reasons for this sluggish adoption of these methods may well be that they require the use of non-linear solvers, which are not available or hard to use in standard applied econometrics software.

We therefore develop a simple estimation procedure to perform structural gravity equation estimation, including the performance of counterfactual analysis. Our development rests on the shoulders of the theoretical developments of Anderson (1979) and Anderson and van Wincoop (2003) and the suggested estimation of structural gravity models by Santos Silva and Tenreyro $(2006)$ and the investigated properties of this estimator by Fally $(2015)$. Specifically, we exploit the combined properties of the theory and the properties of the suggested Poisson Pseudo-Maximum-Likelihood estimator to develop a (constrained) estimation approach that allows us to estimate conditional and full general equilibrium responses to changes in trade costs.

We apply our suggested estimation procedure to a hypothetical scenario that removes all international borders to trade in manufactures for 40 countries and a rest-of-world aggregate over the period 1990-2002. Our results suggest that abolishing international borders leads to welfare gains between about 5 and 40 percent when accounting for changes in multilateral resistance terms and general equilibrium effects via changes in output and expenditure. We also compare these results with ones obtained by solving the non-linear equation system, leading to identical results.

We also highlight how our framework can be extended beyond the endowment economy. We hope that this discussion highlights how easily our approach can be generalized and, combined with the fact that only a standard econometric software such as Stata is necessary 
to use our approach, will open up the door to the application of the most recent developments in trade policy analysis to the large group of applied economists and policymakers. 


\section{References}

Allen, Treb, Costas Arkolakis, and Yuta Takahashi. 2014. "Universal Gravity." unpublished manuscript, available for download at http://www.econ.yale.edu/ ka265/research.html.

Anderson, James E. 1979. "A Theoretical Foundation for the Gravity Equation." American Economic Review, 69(1): 106-116.

Anderson, James E. 2011. "The Gravity Model." Annual Review of Economics, 3: 133-160.

Anderson, James E., and Eric van Wincoop. 2003. "Gravity with Gravitas: A Solution to the Border Puzzle." American Economic Review, 93(1): 170-192.

Anderson, James E., Mario Larch, and Yoto V. Yotov. 2015. "Growth and Trade with Frictions: A Structural Estimation Framework." NBER Working Paper No. $2137 \%$.

Anderson, J.E., and Yoto V. Yotov. 2010. "The Changing Incidence of Geography." American Economic Review, 100(5): 2157-2186.

Anderson, J.E., and Yoto V. Yotov. 2015. "Terms of Trade and Global Efficiency Effects of Free Trade Agreements, 1990-2002." Journal of International Economics, forthcoming, doi:10.1016/j.jinteco.2015.10.006.

Arkolakis, Costas, Arnaud Costinot, and Andrés Rodríguez-Clare. 2012. "New Trade Models, Same Old Gains?" American Economic Review, 102(1): 94-130.

Armington, P.S. 1969. "A Theory of Demand for Products Distinguished by Place of Production." IMF Staff Papers, 16: 159-176.

Caliendo, Lorenzo, and Fernando Parro. 2015. "Estimates of the Trade and Welfare Effects of NAFTA." Review of Economic Studies, 82(1): 1-44.

Costinot, Arnaud, and Andrés Rodríguez-Clare. 2014. "Trade Theory with Numbers: Quantifying the Consequences of Globalization." Chapter 4 in the Handbook of International Economics Vol. 4, eds. Gita Gopinath, Elhanan Helpman, and Kenneth S. Rogoff, Elsevier Ltd., Oxford.

Dekle, Robert, Jonathan Eaton, and Samuel Kortum. 2008. "Global Rebalancing with Gravity: Measuring the Burden of Adjustment." IMF Staff Papers, 55(3): 511-540.

Eaton, Jonathan, and Samuel Kortum. 2002. "Technology, Geography and Trade." Econometrica, 70(5): 1741-1779.

Fally, Thibault. 2015. "Structural Gravity and Fixed Effects." Journal of International Economics, 97(1): 76-85.

Feenstra, R.C. 2004. Advanced International Trade: Theory and Evidence. Princeton, New Jersey:Princeton University Press.

Fernández-Valz, Iván, and Martin Weidner. 2015. "Individual and Time Effects in Nonlinear Panel Models with Large N, T." unpublished manuscript available for download at http://arxiv.org/pdf/1311.7065v3.pdf. 
Head, Keith, and Thierry Mayer. 2014. "Gravity Equations: Workhorse, Toolkit, and Cookbook." Chapter 3 in the Handbook of International Economics Vol. 4, eds. Gita Gopinath, Elhanan Helpman, and Kenneth S. Rogoff, Elsevier Ltd., Oxford.

Mathworks. 2013. "Matlab: Release 2013b." 3 Apple Hill Drive, Natick, MA 01760-2098, United States, http://www.mathworks.com/.

Santos Silva, João M.C., and Silvana Tenreyro. 2006. "The Log of Gravity." Review of Economics and Statistics, 88(4): 641-658.

Santos Silva, João M.C., and Silvana Tenreyro. 2011. "Further Simulation Evidence on the Performance of the Poisson Pseudo-Maximum Likelihood Estimator." Economics Letters, 112(2): $220-222$.

StataCorp LP. 2013. "Stata Statistical Software: Release 13.1." College Station, Texas 77845 USA, http://www.stata.com/.

Waugh, Michael E. 2010. "International Trade and Income Differences." American Economic Review, 100(5): 2093-2124. 

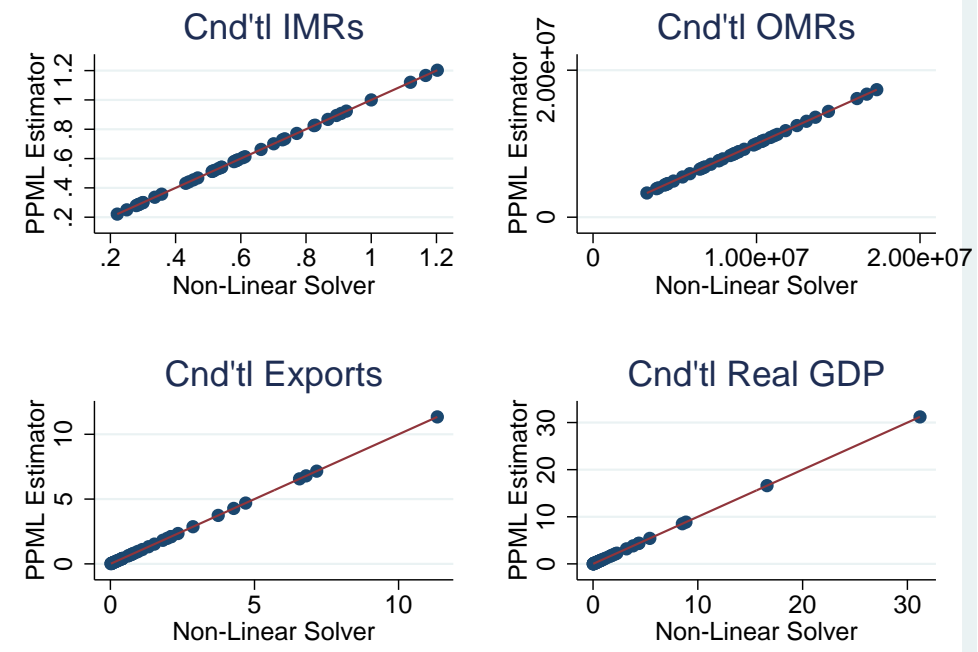

Note: These figures compare the results from Matlab and Stata for (clockwise, starting from the upper left) the IMR, OMR, the real GDP (in $100 \mathrm{~m}$ dollars), and total exports (in $100 \mathrm{~m}$ dollars) of each country for the 'Conditional' GE effects when abandoning international borders.

Figure 1: 'Conditional' GE Results: Matlab versus Stata
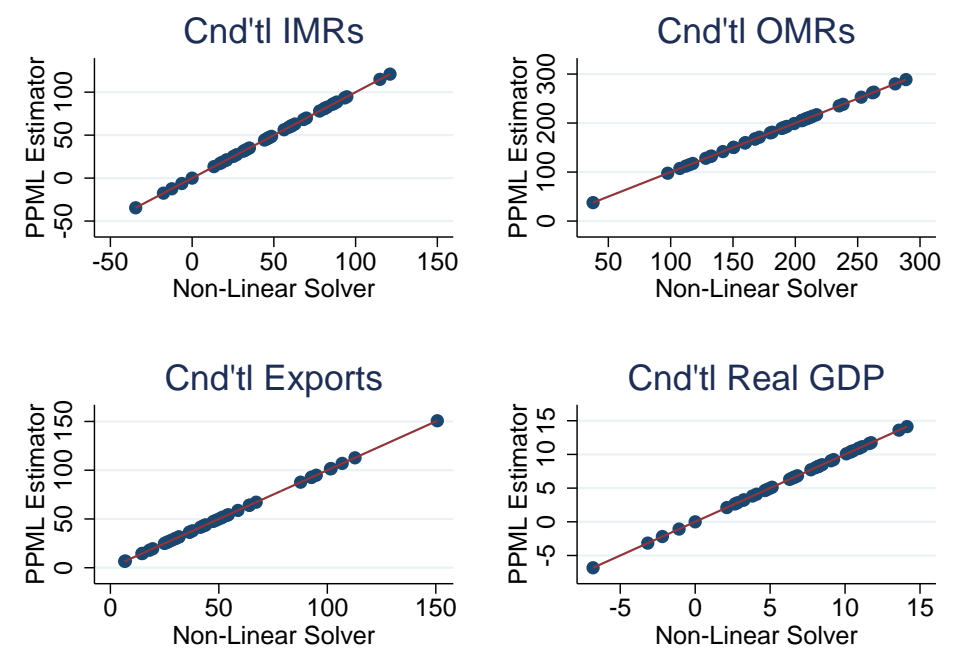

Note: These figures compare the results from Matlab and Stata for (clockwise, starting from the upper left) the changes (in percent) of the IMR, the changes (in percent) of the OMR, welfare effects in percent (calculated as changes in real GDP), and the changes (in percent) of total exports of each country for the 'Conditional' GE effects when abandoning international borders.

Figure 2: 'Conditional' GE Indexes: Matlab versus Stata 


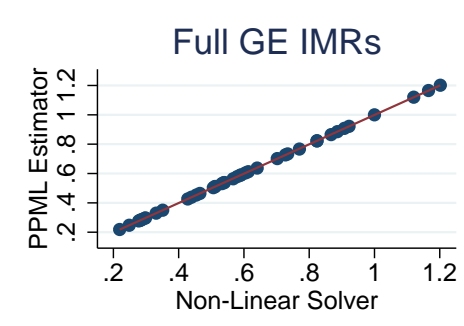

Full GE Exports

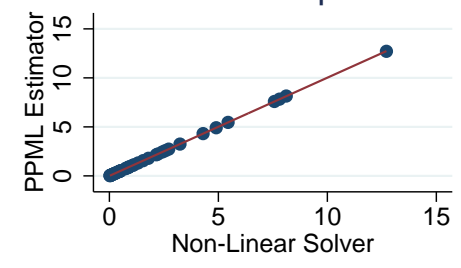

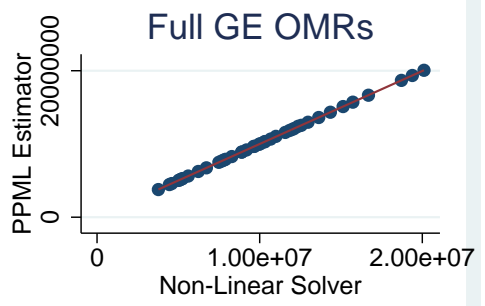

Full GE Real GDP

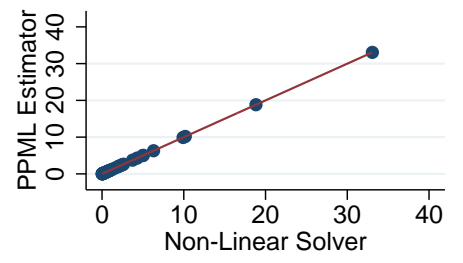

Note: These figures compare the results from Matlab and Stata for (clockwise, starting from the upper left) the IMR, OMR, the real GDP (in $100 \mathrm{~m}$ dollars), and total exports (in $100 \mathrm{~m}$ dollars) of each country for the 'Full Endowment' GE effects when abandoning international borders.

Figure 3: 'Full Endowment' GE Results: Matlab versus Stata

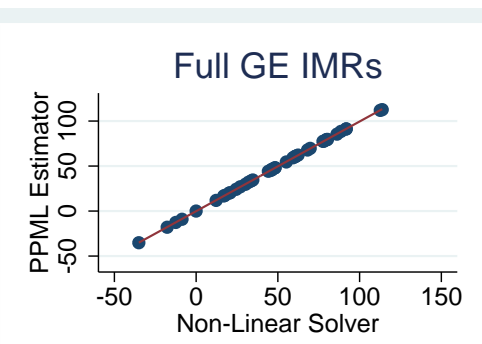

Full GE Exports

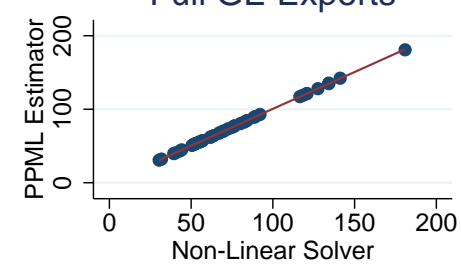

Full GE OMRs

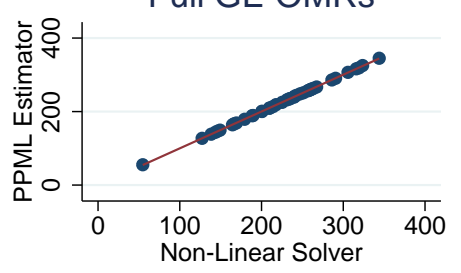

Full GE Real GDP

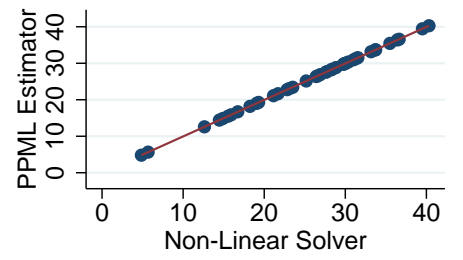

Note: These figures compare the results from Matlab and Stata for (clockwise, starting from the upper left) the changes (in percent) of the IMR, the changes (in percent) of the OMR, welfare effects in percent (calculated as changes in real GDP), and the changes (in percent) of total exports of each country for the 'Full Endowment' GE effects when abandoning international borders.

Figure 4: 'Full Endowment' GE Indexes: Matlab versus Stata 


\section{Appendix: Implementation in Stata}

This Appendix provides the Sata code that corresponds to the three main steps from our procedure. The actual Stata 'data' and 'do' files used to obtain the GE indexes from the main text are available by request.

\section{Stata Commands}

(

qui tab exporter

local $\mathrm{NoC}=r(r)$

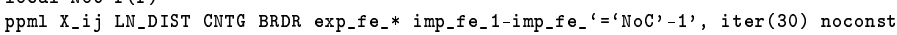

predict hat_X_ij, mu

scalar DIST_est=_b[LN_DIST]

scalar CNTG_est=_b[CNTG]

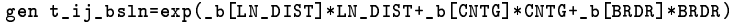

gen $t_{-} i j_{-} \operatorname{ctrf}=\exp \left(\_b[\right.$ LN_DIST] $*$ LN_DIST+_b $[\mathrm{CNTG}] * \mathrm{CNTG}+$ _b $[\mathrm{BRDR}] * \mathrm{BRDR} * 0)$

$* * * * * * * * * * * * * * * * * * * * * * * * * * * * * * * * * * * * * * * * * * *$

* Step 1.b: Construct 'Baseline' GE Indexes*

$* * * * * * * * * * * * * * * * * * * * * * * * * * * * * * *, 1)$

forvalues $i=1(1)$ ' $=$ ' $N$ NoC' -1 ' \{

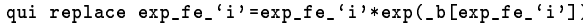

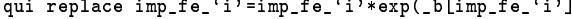

\}

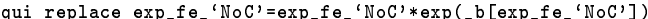

qui replace imp_fe ' $\mathrm{NoC}$ ' $=i m p_{-} \mathrm{fe}_{-}{ }^{\prime} \mathrm{NoC}$ ' $* \exp (0)$

egen all_exp_fes_o=rowtotal (exp_fe_ $1-\exp _{-} f e_{-}(=' N o C$ ')

egen all_imp_fes_o=rowtotal (imp_fe_1-imp-fe $e_{-}(=' N o C '$ )

gen omr_bsln=Y $\mathrm{Y}_{-} i * E_{-} d e u /\left(a l l_{-} e x p_{-} f e s_{-} 0\right.$ )

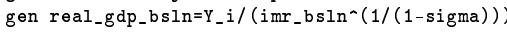

* Step 2.a: Estimate 'Conditional, Gravity

drop exp_fe_* imp_fe_*

qui tab exporter, gen (exp_fe_)

qui tab importer, gen (imp_fe-

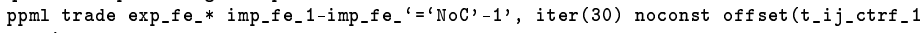

predict trade_cndl, mu
* Step 1.a: Estimate 'Baseline' Gravity*

gen $t_{-} i j_{-} \operatorname{ctrf} f_{-} 1=\log \left(t_{-} i_{j}\right.$ ctrf $)$

gen $i m r_{-} b s l n=E_{-} j /\left(a l l_{-} i m p \_f e s_{-} 0 * E_{-} d e u\right)$

\section{Comment/Description}

*Obtain number of countries

* Obtain trade cost elasticities using Eq. (9) *Save predicted trade.

* Save estimates of trade cost elasticities.

*Construct baseline $t_{-i j \text {-(1-sigma) }}$

*Construct counterfactual $t_{-} i j^{\wedge}$ (1-sigma)

* Combine estimates of fixed effects

*Construct OMRs from Eq. (7)

*Construct IMRs from Eq. (8)

*Construct Real GDP.

*Drop estimated FEs as they will be updated. *Create new FEs.

* Obtain 'Conditional' FEs from Eq. (11) *Save predicted trade for Step 3 .

* Step 2.b: Repeat Step 1.b with the new fixed effects and original trade data to obtain the 'Conditional' GE indexes of interest.

$* * * * * * * * * * * * * * * * * * * * * * * * * * * * * * * * * * * * * * * * * * * * * *)$

* Step 3.a: Estimate 'Full Endowment' Gravity*

scalar sigma $=7$

local $i=3$

local diff_all_exp_fes_sd $=1$

local diff_all_exp_fes_max $=1$

while ('diff_all_exp_fes_sd'>0.01) | ('diff_all_exp_fes_max'>0.01)

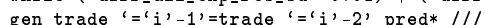

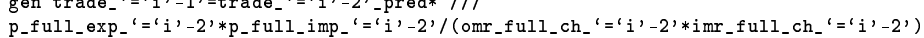

drop exp_f $e_{-} *$ imp_f $e_{-} *$

qui tab exporter, gen (exp_fe $)$

qui tab importer, gen (imp_fe_)

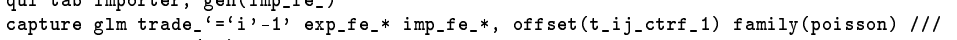

noconst irls iter(30)

predict trade $'$ ' $' i{ }^{\prime}-1$ ' -pred, mu

forvalues $j=1$ (1) ' $\mathrm{NoC}$ '

qui replace exp_fe $f e_{-} j=\exp f e_{-}(j) * \exp \left({ }_{-} b\left[\exp f e_{-}(j],\right)\right.$

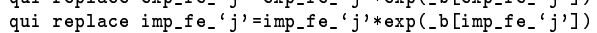
\}

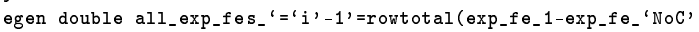

egen double all_imp_fes $\left\{=\left(i,-1\right.\right.$ '=rowtotal (imp_fe_1-imp_fe $e_{-}$'NoC')

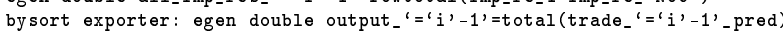

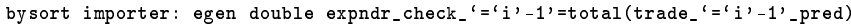

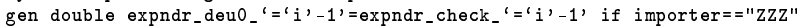

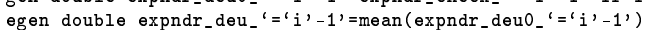

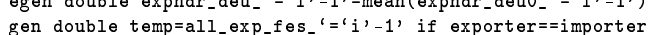

gen double temp=all_exp_fest importer: egen double all_exp_fes $s_{-}\left(={ }^{\prime} i{ }_{-1},{ }_{-} i m p=m e a n\right.$ (temp)

drop temp*

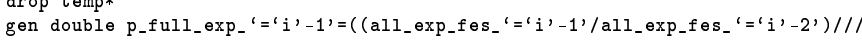

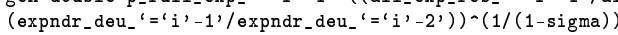

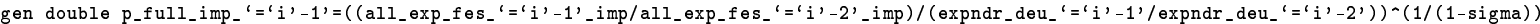

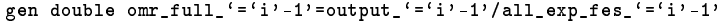

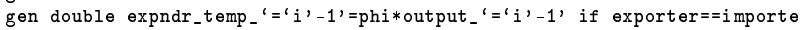

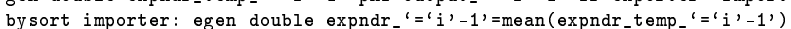

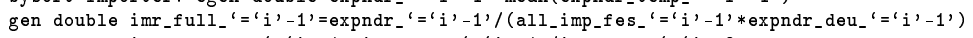

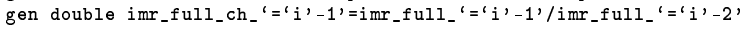

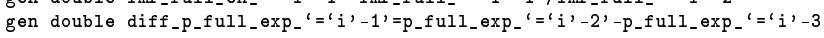

sum diff_p_full_exp_' $=$ ‘ $i{ }_{-1}$ '

local diff_all_exp_fes_sd=r(sd)

local diff_all_exp_fes_max $=a b s(r(\max ))$

local $i=' i ’+1$

\}

* Step 3.b: Repeat Step 1.b with the new fixed effects and the new trade values to obtain the 'Full Endowment, GE indexes of interest. $*$

*Update trade according to Eq. (14).

*Drop estimated FEs as they will be updated.

*Estimate Eq. (11) with new trade values.

*Combine updated estimates of fixed effects.

*Update output

*Update expenditure

*Update factory-gate prices according to Eq. (15)

*Equation (7)

*Update expenditure

*Equation (8)

*Convergence criteria in terms of changes

in factory-gate prices 